\title{
Les pionniers n'ont jamais la tâche facile
}

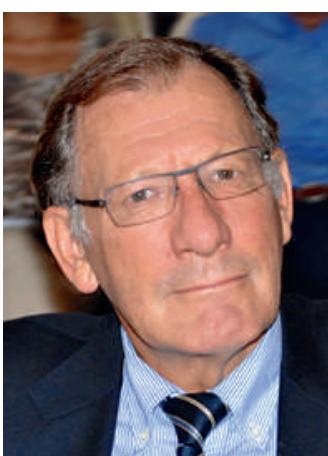

Jean Martin
Le dernier bulletin de l'ASSM [1] est consacré aux soins palliatifs, aujourd'hui promus par l'OMS, une stratégie nationale suisse et des programmes cantonaux. Sujet très important dans une société où moins de $20 \%$ des personnes ont la chance de mourir à domicile alors que, avec un niveau adéquat de tels soins, ce pourrait être $80 \%$. Pourtant, le nécessaire changement de paradigme et de mentalité se fait très lentement. «L'acharnement thérapeutique en fin de vie est toujours fréquent, ce qui représente un problème autant éthique que pratique», dit le professeur lausannois G. B. Borasio.

En France, Didier Sicard, ancien président du Comité national d'éthique, a dirigé une commission qui a publié à fin 2012 un rapport sur la fin de vie. Ses conclusions peuvent être dites dévastatrices: «Nos auditions ont mis au jour le malaise, voire la colère des citoyens; de nombreuses situations de fin de vie ne sont pas correctement appréhendées (...) La recommandation première est de donner la plus grande importance aux paroles et aux souhaits des malades en fin de vie.» En effet, malgré les changements entérinés quant au principe, par la loi Leonetti de 2005 notamment, le corps médical traîne massivement les pieds (voir aussi [3]) pour adopter des pratiques qui amélioreraient clairement la qualité de la vie dans sa dernière phase - tout en la prolongeant de plusieurs mois, par rapport à l'acharnement, dans des études randomisées. Aux Etats-Unis, pourtant très sensibles à l'autonomie du malade, les médecins continuent souvent à refuser de dire au patient que le pronostic est mauvais et qu'il va mourir. A Lausanne le 6 juin dernier, c'est ce que disait encore Joan Tronto, de Minneapolis, spécialiste de l'éthique du soin (care ethics).

J'y pensais en lisant l'autobiographie [4] de notre compatriote Elisabeth Kübler-Ross (1926-2004), mondialement connue pour s'être battue avec courage et persévérance pour une approche de la mort plus ouverte et plus humaine. Elle a marqué par sa description de cinq stades chez la personne en fin de vie: déni, colère, marchandage, dépression, acceptation. Ce livre est très intéressant (surtout pour nous Suisses je trouve): histoire d'une jeune femme (une de trois triplées!) dans une famille zurichoise propre en ordre, avec ce que cela représente (oui!) de sérieux et de travail. Qui s'engage comme volontaire à la fin de la Deuxième Guerre mondiale dans des régions dévastées (France, Pologne), fait un apprentissage de laborantine puis devient médecin. Emigre aux Etats-
Unis, devient psychiatre un peu par hasard et pratique son métier en mettant un accent déterminant sur le contact, l'écoute, l'empathie. Elle travaille avec des mourants et mène avec eux des séminaires à succès suscitant de la part de confrères des critiques selon quoi elle «exploite» les patients. S'intéresse ensuite beaucoup aux expériences de mort imminente.

L'émergence du sida au début des années 1980 la trouve prête à travailler avec ces malades irrémédiablement condamnés à l'époque. Entre autres, elle cherche à ouvrir un centre de traitement pour enfants séropositifs en Virginie mais se heurte à l'opposition féroce d'«un voisinage de bon chrétiens» (on finit par mettre le feu à sa maison). Elle surprend en montrant un intérêt actif pour des pratiques qui sortent du cadre médical usuel, notamment le «channeling» où des guides de type médiums établissent une communication entre un humain et une entité appartenant à une autre dimension (sorte de spiritisme à dimension spirituelle). Cela a pu la rendre «suspecte». Tout en n'ayant pas (J. M.) la moindre compétence dans ce domaine, je suis certain que cela n'autorise pas à la discréditer; elle a été une pionnière remarquable. Citations en passant: «Les enfants mourants, plus que les adultes, vous disent exactement ce dont ils ont besoin pour être en paix.» «Dans les ateliers, les participants parvenaient à se défaire de leur unfinished business (un point sur lequel elle insiste), de la colère accumulée, et à apprendre à vivre de manière à être prêts à mourir à n'importe quel âge.» «Il n'y a pas de problème qui ne soit pas en réalité un cadeau et une opportunité de croissance.» «Beaucoup de choses dans la vie correspondent à trouver ce que vous savez déjà.»

On sait que la grandeur des pionniers, dans tous les domaines, ne leur est souvent accordée qu'à titre posthume. Dans certaines pages de Kübler-Ross, on pense à Ignaz Semmelweiss, rendu fou par le refus de la Vienne médicale du milieu du XIX ${ }^{\mathrm{e}}$ siècle d'ouvrir les yeux sur la réalité qu'elle tuait beaucoup de parturientes en passant la fièvre puerpérale de l'une à l'autre. Nous n'en sommes pas là aujourd'hui; néanmoins, la difficulté persistante de trop de professionnels à «accepter la défaite» et à œuvrer avec le patient pour que sa fin soit la plus libre de douleur, sereine et riche de relations, est un problème.

Jean Martin, membre de la

Commission nationale d'éthique et de la rédaction

\begin{abstract}
Comission nationale deethique et de la redaction
\end{abstract}

